\title{
QOS of WIMAX Interworking with WLAN to Supply Harmonious Services
}

\author{
Salma Mohmed Abdalh Mohamed ${ }^{1}$, Dr. Mohmmed Abakar ${ }^{2}$ \\ Department of Communication Engineering, Al-Neelain University
}

\begin{abstract}
Intersystem handover with ongoing mobility management between the network is a serious issue for operators to focus on. Therefore the proposed model represents an intersystem handover algorithm between WIMAX and WLAN. The presented Network model will achieve unified mobile and for cellular and broadband wireless access coverage. Here coupling methods like loose coupling scheme and tight coupling scheme in the interworking also discussed and the results were compared. By having a method with MIPV4 implementation. the intersystem handover issue can be resolved. The approach of this model is to reduce the packet loss during handover. As a result the end user can get services continuously with minimum packet loss.
\end{abstract}

Keywords: WLAN, WIMAX, Vertical Handover, MIPv4, OPNET, Loose Coupling, Tight Coupling

\section{Introduction}

The motivations of $4 \mathrm{G}$ networks is the ubiquitous wireless access abilities which provide the seamless handover for the end users in the heterogeneous networks of different access technologies. All the available technologies have some advantages and disadvantages. These technologies differ in terms of spectrums. bandwidths. media access technologies. security mechanisms and so on. The well established $3 \mathrm{G}$ network like WIMAX can provide high mobility with wide area coverage but can support low to medium data rate which is not sufficient to satisfy data-intensive applications and the service charge is also very high. WIMAX is not suitable for small. indoor and densely populated areas. The recently invented WLAN(IEEE 802.11g) was standardized to support mobility to the end user with wider coverage and faster speed. The salient features of the Mobile WLAN (IEEE 802.16e) are high data rates up to $63 \mathrm{Mbps}$ for DL and $28 \mathrm{Mbps}$ for UL. QoS. scalability. security and mobility supporting handover schemes with latencies less than $50 \mathrm{~ms}$ . Also the deployment cost of the Mobile WLAN (IEEE $802.11 \mathrm{~g}$ ) is very low. The average $3 \mathrm{G}$ spectrum cost/Hz is 1000 times higher than the average WLAN spectrum $\operatorname{cost} / \mathrm{Hz}$ in Europe. But the vital drawback of the WLAN is that the standard does not fully define the complete network infrastructure. So it is still a far future to deploy the WLAN network of its own. But it can be interworked with the existing networks.

The vertical handoff process between different existing networks in the wireless communication has become one of biggest challenge for the operators. The interworking between WLAN and WIMAX can be useful to get the benefits from both the systems and the handover between these two networks will make the end user connected whenever. wherever and for any services. The rest of the paper is organized as follows: Firstly our proposed method of Loose coupling. tight coupling and handover algorithm is described briefly. The simulation environment built by the OPNET Modeler 14.5 and the results are described in the third section and finally we abrogate with a short concluding remarks followed by the acknowledgement of the work.

\section{A. Interworking Architecture}

The most critical elements to be resolved for intersystem handover area)avoid the change of IP. Henceforth. Mobile IP is used as a solution of the network layer issue.

The next issues are maximum packet loss during handover. To minimize packet loss the preferred type of handover would be soft handover. But to achieve soft handover in different network environment . the mobile device must have multiple interfaces to communicate with the different networks at the same time as needed for soft handover. Hence. the mobile device assumed to have two transceivers; one for WLAN interface and one for WIMAX interface. The mobile device is designed such that it has the intelligence to monitor the signal strength and can make the decisions of handover. Hence. the handover is mobile initiated.

The next issue is the version of IP to be used. 3GPP IMS is mainly designed for IPv6 but according to the early releases IMS can also be implemented using the IPv4. The WLAN can support both IPv4 and IPv6 but the WIMAX SGSN does not support the IPv6. Hence. if IPv6 is used there will be an additional latency regarding to the NAT every time passing through the SGSN. But if IPv4 is used for IMS.

The proposed overall network. as shown in Fig. 1, shows the components of MIP. The main components of MIP are the foreign agent (FA) and the home agent (HA). 


\section{International Journal of Science and Research (IJSR) \\ ISSN (Online): 2319-7064}

Index Copernicus Value (2013): 6.14 | Impact Factor (2015): 6.391

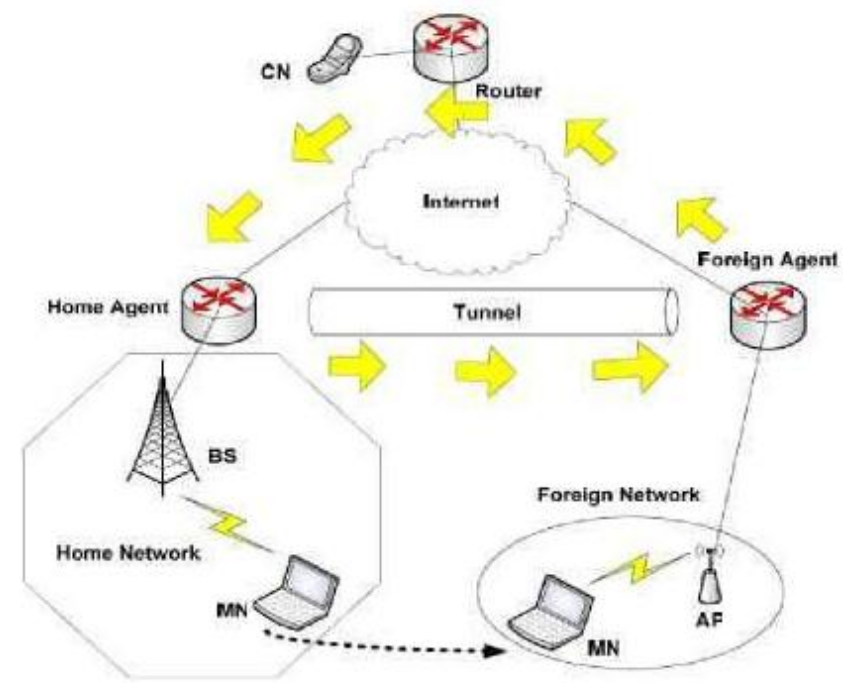

Fig 1 Components of MIP

In WIMAX networks GGSN acts as a home agent since MN initially in the WIMAX network and in WLAN network router acts as a foreign agent. The server bandwidth is distributed among the network according to the speed to be supported and server channel frequency is kept less than the channel frequency of the base station so that there remains no in-transit packet in queue during the handover to eliminate the packet loss in the radio path.

\section{B. Mobile IPV4}

Mobile IP's is the standard protocol defined for terminal mobility in the Internet; therefore, Mobile IP is proposed as the basic mechanism to provide service continuity. Mobile IP is the key protocol to enable mobile computing and networking, which brings together two of the world's most powerful technologies, the Internet and mobile communication. In Mobile IP, two IP addresses are provided for each computer: home IP address which is fixed and care- of IP address which is changing as the computer moves. When the mobile moves to a new location, it must send its new address to an agent at home so that the agent can tunnel all communications to its new address timely. The article limits its scope to Mobile IPv4, Mobile IP for IP version 4 .

The Internet Protocol version 4 (IPv4) is a fundamental network layer protocol that contains addressing information and some control information that enables data packets to berouted. Mobile IPv4 can provide transparency to the upper layers while providing seamless mobility using the care-of addresses. It is noted that Mobile IP provides service continuity, and seamless service continuity can be provided using standardized extensions of the base protocol such as Fast handover. Mobile IP requires the deployment of home agents and a protocol between the mobile nodes, home agent, and corresponding nodes. The home agent placement and home address assignment issues are addressed for supporting Mobile IP for heterogeneous roaming. The main objectives of this paper are to integrate WLAN with cellular network using different architecture and to analyze the performance of the proposed architecture.

\section{Interworked WLAN / 3G Cellular Networks}

When a MN moves from the WIMAX coverages area into the WLAN network as depicted in Figure 8, the VHO procedure occurs. Specifically, the MN of the WIMAX network first scans to ensure that a WLAN network access within the vicinity is available. The cellular WIMAX/BS periodically transmits a CoA advertisement for the outgoing MNs to WLAN networks to track the availability of the WIMAX network. When the MN detects the advertisement, it begins examining the received signal strength (RSS) to make the VHO decision. The MN first estimates the RSS from the WLAN access router and compares it with the WIMAX network. If the RSS of the WLAN is found to be greater than the WIMAX, then the VHO procedure is initiated. Otherwise, the MN maintains its connectivity with the WIMAX network. The RSS of the WIMAX will continue to get weaker as the MN moves away from the WIMAX/BS towards the WLAN/AP. As the MN approaches the overlapping area of two networks, it informs the $\mathrm{CN}$ to start the bicasting of data packets via both WIMAX-IP1 and WLAN-IP2.As the movement towards the WLAN network intensifies, the MN senses the high RSS of the new link and transmits a registration request to the access router of the cellular WIMAX. It then assigns a new CoA from the old access router to the new WLAN-IP2 network. The WLAN HA then begins to track the location of the MN during the course of the in MIP. Similarly, when the MN moves into the coverage area of the cellular WIMAX, it begins a reverse VHO procedure. VHO procedure is clearly explained in Fig $2^{\prime}$

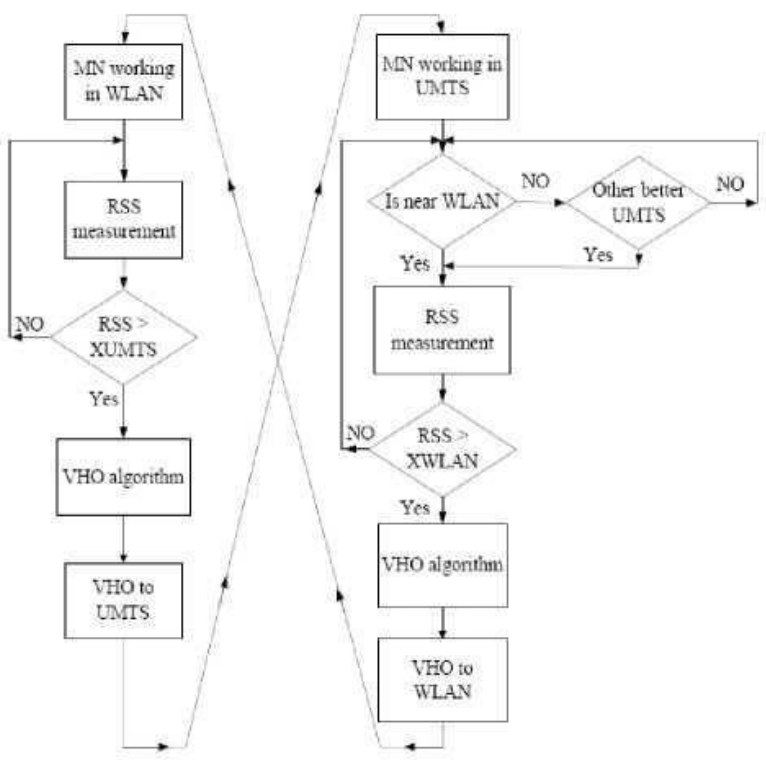

Figure 2: Vertical Handover Algorithm

\section{Loose Coupling}

In the loose coupling architecture both networks interconnect independently and utilizing one common subscription. They provide independent services. In loose coupling the interworking point is after the interface point of GGSN (Gateway GPRS Node) with the IP network and the network interconnection uses MIP mechanism for mobility between WLAN and WIMAX. it has no direct link to $3 \mathrm{G}$ network equipment. As a result the data traffic from WLAN 


\section{International Journal of Science and Research (IJSR) \\ ISSN (Online): 2319-7064}

Index Copernicus Value (2013): 6.14 | Impact Factor (2015): 6.391

goes through the internet and does not pass through WIMAX core network. In case of third party network mobility and roaming is enabled via dedicated connections between the operator and wireless LAN. The WLAN gateway must support MIP functionalities for handling mobility access the different networks. The one advantage of using loose coupling is it allows independent deployment $\&$ traffic engineering of heterogeneous network. It also enables $3 \mathrm{G}$ operators without major investment to take advantage of WLAN.

\section{E. Tight coupling}

In a tight coupling scenarios the WLAN are directly connected with 3G(WIMAX)core network as in any other radio access network (RAN).In this context WLAN can execute functions that are available in the $3 G(R A N)$. The WLAN gateway will introduce to achieve integration and hides the detail of its own network to 3G/WIMAX network. All $3 \mathrm{G}$ protocol is implemented(mobility management ,authentication etc).As a result the data from WLAN user will goes through 3G/WIMAX core network then to internet or packet data network PND. In this context each network will modify their protocol interfaces and services for supporting interworking requirement. In a tight coupling the interconnection with WLAN can be made at a core level (GGSN, SGSN) or Access level (RNC).

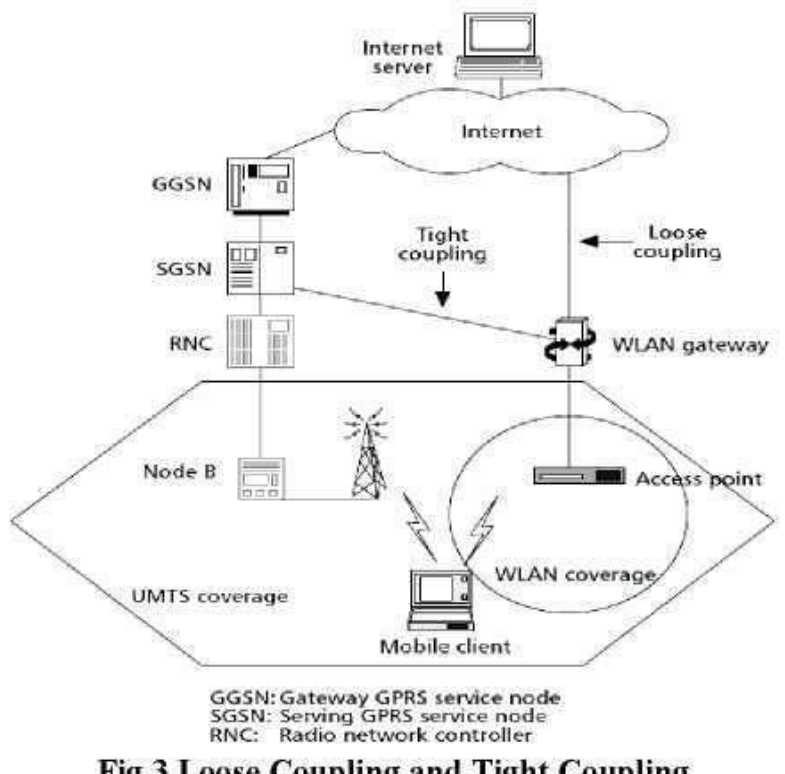

\section{Results and Performance Analysis}

We have implemented our proposed method described in the previous section by the OPNET Modeler 14.5 and carried out the simulations to characterize the performance of the proposed method of intersystem handover.

\section{A. Simulation Modele}

We have designed two different networks for scenarios which are loose coupling scheme and tight coupling scheme for interworking between WLAN and WIMAX. Network design for the two scenarios is shown in the figures below.

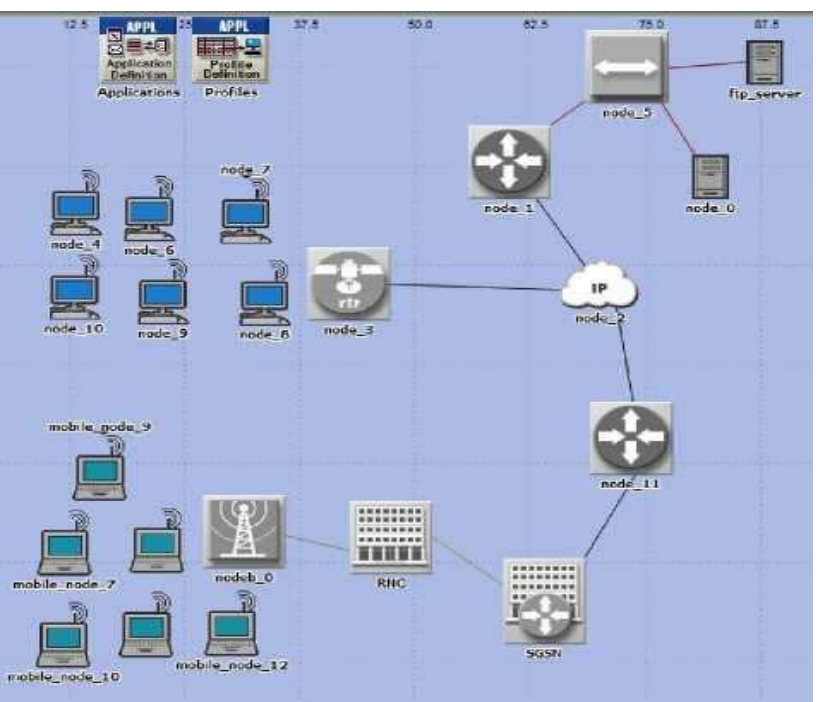

Figure 4: Loose Coupling

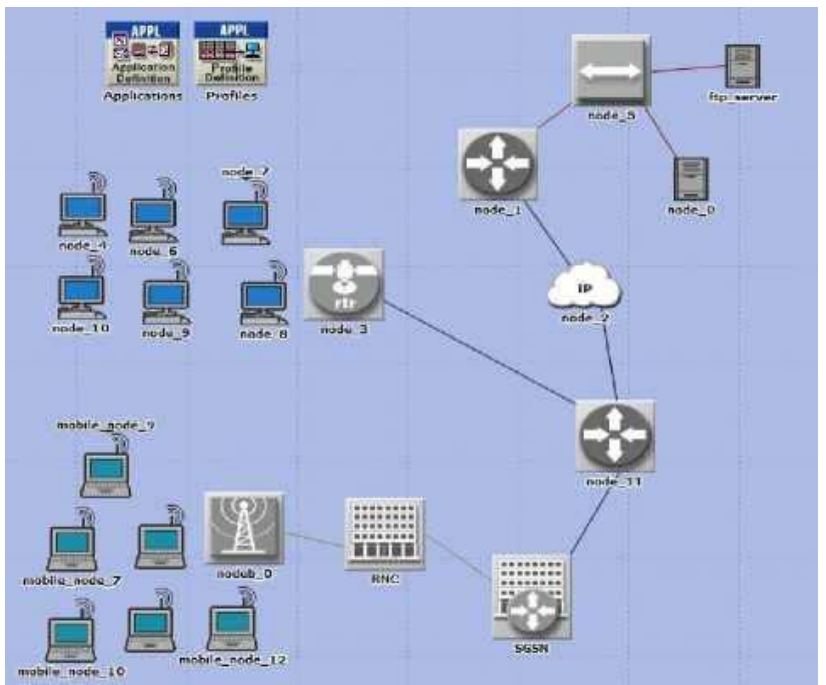

Figure 5: Tight Coupling

\section{B. Simulation Results}

The simulation was performed for FTP session and HTTP session.FTP Uploade and download response time, HTTP page response time results were analyzed.

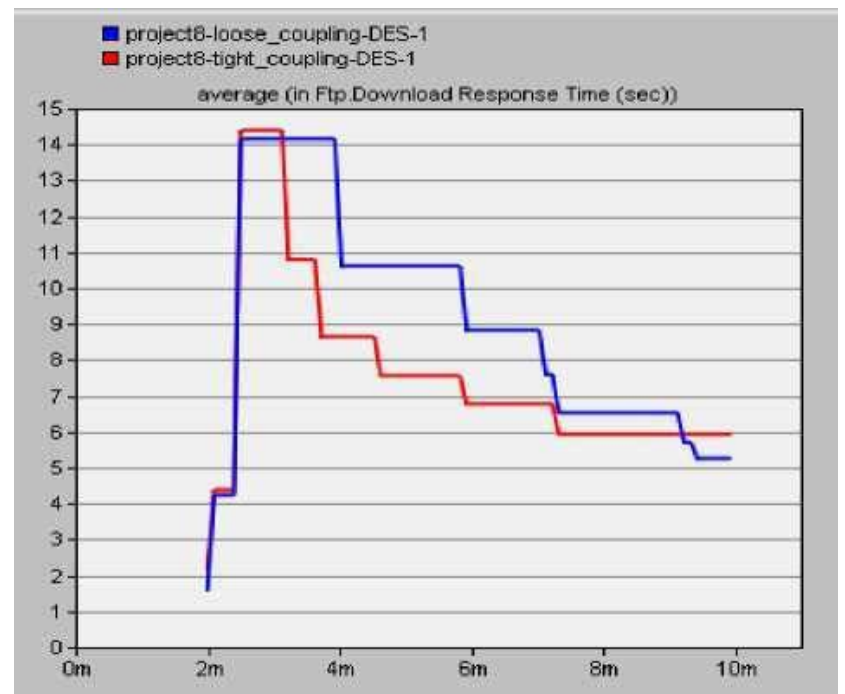

Figure 6: FTP Download Response Time

Volume 5 Issue 6, June 2016 www.ijsr.net 


\section{International Journal of Science and Research (IJSR) \\ ISSN (Online): 2319-7064 \\ Index Copernicus Value (2013): 6.14 | Impact Factor (2015): 6.391}

Fig 6 shows that loose coupling scheme takes response time more than tight coupling scheme. As the time increases the response time gradually decreases. It is clear from that users move from WIMAX to WLAN.WLAN has higher bandwidth than WIMAX. So users take less download response time for FTP.

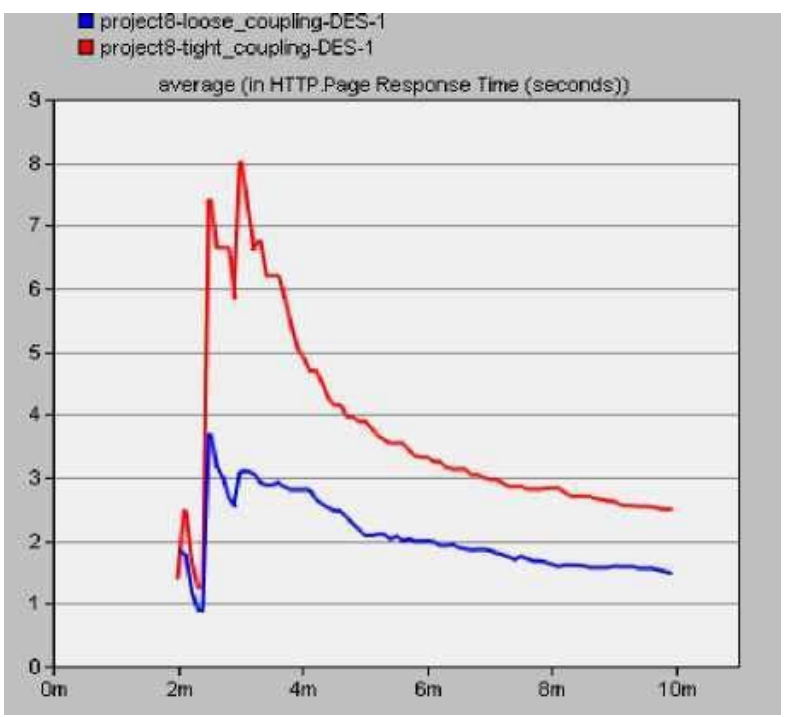

Figure 7: HTTP Page Response Time

Fig 7 shows that initially users take more response time, as the time gradually increases HTTP page response time decreases it is clear from that users move from WIMAX to WLAN. Here loose coupling is better than tight coupling for HTTP.

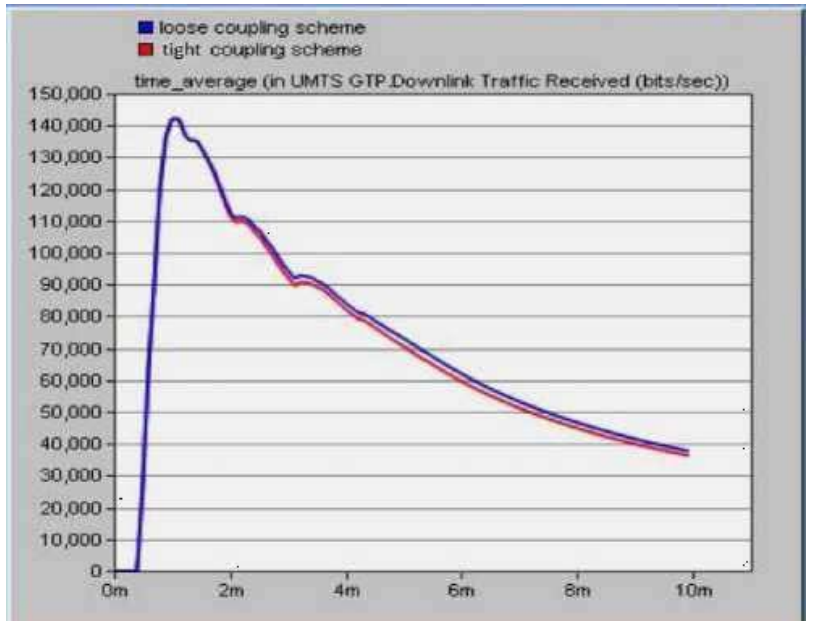

Figure 8: Downlink Traffic Received

Fig 8 shows the graphs of traffic sent and traffic received In WIMAX network while the users start moving from WIMAX network to WLAN network, as users move to WLAN network very few people left in WIMAX network and results in lower data sent and received and if we compare loose coupling to the tight coupling data sent and received for WIMAX network is higher in loose coupling architecture.
Response time (seconds) for user applications in WIMAX and WLAN networks.

\begin{tabular}{|c|c|c|c|l|}
\hline $\begin{array}{c}\text { Application } \\
\mathrm{s}\end{array}$ & Minimum & $\begin{array}{c}\text { Maximu } \\
\mathrm{m}\end{array}$ & $\begin{array}{c}\text { Averag } \\
\mathrm{e}\end{array}$ & Network \\
\hline HTTP & 0.909 & 1.755 & 1.268 & UMTS \\
\hline FTP & 7.094 & 14.347 & 7.071 & UMTS \\
\hline HTTP & 0.013 & 0.049 & 0.032 & WLAN \\
\hline FTP & 0.775 & 3.657 & 0.51 & WLAN \\
\hline
\end{tabular}

\section{Conclusions}

In this article we described the integration model for WIMAX AND Wireless LAN .And we have explained two types of integration models between the WIMAX and WLAN(loose coupling architecture ,tight coupling architecture ).We have discussed here in this chapter the benefits an drawbacks of integration models. we have performed the simulation by using OPNET 14.5 as a simulation tool. We have compared the two integration schemes (loose coupling architecture, tight coupling architecture) our results shows that loose coupling architecture is better than the tight coupling architecture scheme. Our results shows that response time for FTP, HTTP is smaller in loose coupling architecture scheme as compare to the tight coupling scheme. We have also computed user movement between two different technology networks, there will be some packet loss and session interruption when the user move between two different networks .this can be resolved by implementing 3 GPP SIPIMS model. By implementing SIP-IMS model end user can get the services continuously without any interruption. Implementation of SIP-IMS model and maintenance of the session management is the future work.

\section{References}

[1] THEODORE S RAPPAPORT — Wireless Communications Principles and practices! 2nd edition

[2] Lane Pool - Cellular Communication Explained From Basic to 3G!

[3] LaMaire, R.O. et al., -Wireless LANs and Mobile Networking: Standards and Future Directions,! IEEE Communications Magazine, Vol. 34, No. 8,Aug. 1996, pp. 86-94.

[4] Crow, B.P., I. Widjada, J.G.Kim, P.T.Sakai, -IEEE 802.11 Wireless Local Area Networks,! IEEE Communications Magazine, September 1997, pp.116-126.

[5] Chen, K. C., -Medium Access Control of Wireless LANs for Mobile Computing,! IEEE Network, Vol. 8, No. 5, Sept. 1994, pp. 50-63.

[6] Weinmiller, J., H. Woesner, and A. Wolisz, - Analyzing and Improving the IEEE 802.11- MAC Protocol for Wireless LANs,! Proc. MASCOTS '96, San Jose, CA, Feb. 1996, pp. 200-206.

[7] IEEE Std 802.1X-2001 -IEEE Standard for Local and metropolitan area networks-Port- Based Network Access Control,! 14 June 2001.

[8] IEEE P802.11e/D1, March 2001, Draft Supplement to IEE Std 802.11, 1999 Edition, Draft Supplement to STANDARD FOR Telecommunications and Information Exchange Between Systems — LAN/MAN Specific

[9] Blunk, L., J. Vollbrecht, and Bernard Aboba, — Extensible Authentication Protocol (EAP),! October 2002. IETF pppext working group draft draft-ietfpppext- rfc2284bis- 


\section{International Journal of Science and Research (IJSR) \\ ISSN (Online): 2319-7064}

Index Copernicus Value (2013): 6.14 | Impact Factor (2015): 6.391

07.txt. [10] ISO/IEC 8802-11, ANSI/IEEE Std 802.11, First

Edition 1999-00-00, Information Technology

Telecommunications and information exchange between

systems - Local and metropolitan area networks -

Specific requirements - Part 11: Wireless LAN Medium

Access Control (MAC) and Physical Layer (PHY)

specifications

Volume 5 Issue 6, June 2016

www.ijsr.net

Licensed Under Creative Commons Attribution CC BY 